\title{
Factores de riesgo asociados a mortalidad en infecciones relacionadas con la atención en salud en un hospital universitario de tercer nivel en Colombia
}

\author{
Christian José Pallares, Ernesto Martínez \\ Hospital Universitario del Valle "Evaristo García”, E.S.E., Cali, Colombia
}

Introducción. Las infecciones hospitalarias son una amenaza para la salud pública. A pesar de los esfuerzos para contenerlas, su incidencia sigue siendo grande y genera altos costos en la atención en salud.

Objetivo. Determinar los factores asociados a mortalidad en pacientes con diagnóstico de infecciones hospitalarias en nuestra institución.

Materiales y métodos. Se llevó a cabo un estudio prospectivo de cohortes entre enero y diciembre del 2011 por medio de la observación de 1.015 pacientes con diagnóstico de infección de acuerdo a los criterios del sistema de vigilancia hospitalaria sugeridos por los Centers for Disease Control and Prevention (CDC). Se excluyó a quienes no tenían cultivo microbiológico de la infección o habían tenido reingresos hospitalarios en menos de un año. Se evaluaron variables sociodemográficas y clínicas, perfiles de resistencia microbiológica y uso de antibióticos. La variable de desenlace fue la muerte. Se realizó un análisis de supervivencia para cada variable, estableciendo significación estadística con la prueba de log-rank, así como un análisis multivariado mediante regresión de Cox. Se consideraron significativos los valores de $p$ menores de 0,05.

Resultados. El promedio de edad fue de 43 años (57\% hombres y $43 \%$ mujeres); $53 \%$ de los pacientes tuvo diagnóstico clínico y $47 \%$, quirúrgico; $54 \%$ de las infecciones se presentó en la herida quirúrgica y $62 \%$ de ellas se asociaron a microorganismos Gram negativos. La mortalidad durante el seguimiento fue de $24,4 \%$. En el análisis multivariado se encontró asociación con mortalidad para las variables de estancia en cuidado intensivo (hazard ratio $(\mathrm{HR})=1,51 ; \mathrm{IC}_{95 \%} 1,13$ $2,01)$, uso inapropiado de antibióticos $\left(\mathrm{HR}=3,05 ; \mathrm{IC}_{95 \%} 2,34-3,98\right)$ y uso de antibiótico genérico o copia (HR=1,91; IC IC ${ }_{95 \%}$ 1,43-2,55).

Conclusiones. El empleo de moléculas genéricas y el uso inadecuado de antibióticos en pacientes con infecciones hospitalarias son factores que pueden modificarse para disminuir la mortalidad.

Palabras clave: antibacterianos, infección hospitalaria, mortalidad.

doi: http://dx.doi.org/10.7705/biomedica.v34i0.1646

Mortality risk factors associated with healthcare infections in a tertiary level university hospital in Colombia

Introduction: Nosocomial infections are a public health threat. Despite multiple efforts, its incidence is still significant and it generates high costs in health care.

Objective: To determine risk factors associated with mortality in patients with healthcare infections in a tertiary level hospital in Colombia.

Materials and methods: A prospective cohort observational study was performed between January and December 2011. One thousand one hundred and fifteen patients with health care infections using the CDC definition criteria were included. Exclusion criteria were those patients with no microbiologic isolate associated with the infection or hospital readmissions in the last year. Socio-demographic and clinical variables, bacterial resistance profiles and antibiotic use were evaluated. Death was the primary outcome. Survival analysis for each variable was performed using statistical significance defined by the log-rank test. Multivariate and Cox regression analyses were done. Values of $p$ less than 0.05 were considered statistically significant.

Results: Mean age was 43 years old ( $57 \%$ men and $47 \%$ women); $53 \%$ of patients had a medical condition and $47 \%$ surgical diagnosis; $54 \%$ of health care infections were surgical site infections and

\section{Contribución de los autores:}

Los autores contribuyeron en la misma proporción en la elaboración del planteamiento del problema, la definición de la metodología y el tipo de estudio y el establecimiento de objetivos. De igual forma, aportaron a la recolección y elaboración de la base de datos, el análisis estadístico de la información, la redacción de los resultados y la discusión. 
$62 \%$ were associated to Gram-negative bacilli. The mortality rate during follow-up was $24.4 \%$. On multivariate analysis we found an association with intensive care stay ( $\mathrm{HR}=1.51 ; 95 \% \mathrm{Cl}: 1.13-2.01)$, inappropriate use of antibiotics ( $\mathrm{HR}=3.05 ; 95 \% \mathrm{Cl}$ : 2.34-3.98) and use of generic antibiotics or copies (HR=1.91; 95\%Cl: 1.43-2.55).

Conclusions: The use of generic molecules of antibiotics and inappropriate antibiotic treatments in patients with health care infections are modifiable factors to decrease mortality.

Keys words: Anti-bacterial agents, cross infection, mortality.

doi: http://dx.doi.org/10.7705/biomedica.v34i0.1646

Las infecciones asociadas a la atención en salud, también llamadas hospitalarias, son un problema creciente y actualmente se perfilan en el mundo como una amenaza para la salud pública, superando como causa de muerte al VIH, el cáncer de mama o los accidentes de tránsito (1). Como parte de su cuidado médico, la mitad de los pacientes necesitan tratamiento antibiótico debido a esta condición (2), lo cual incrementa los costos de hospitalización, que sólo en Estados Unidos representan entre US $\$ 100$ millones y US $\$ 30.000$ millones anualmente (3), sin tener en cuenta el aumento en la estancia hospitalaria, que puede ir desde cinco hasta 15 días (4-7).

Aunque desde hace más de una década muchos de los esfuerzos de los trabajadores de la salud en el control de infecciones están encaminados a brindar un ambiente hospitalario más seguro para los pacientes, la incidencia de las infecciones asociadas a la atención en salud sigue siendo elevada, con una mortalidad atribuible que puede ser hasta de 35 a $55 \%$, según el tipo de infección $(6,8)$. En la mayoría de las infecciones asociadas a la atención en salud el incremento en la mortalidad está asociado principalmente a la presencia de microorganismos multirresistentes y al tratamiento antibiótico inadecuado (9-13), pero también existen variables propias del paciente (edad, estado nutricional, enfermedades concomitantes) y de la infección (aparición temprana o tardía, tipo, localización y compromiso), que influyen en este desenlace.

El objetivo del presente estudio fue determinar los factores relacionados con la mortalidad en pacientes con diagnóstico de infecciones asociadas a la atención en salud en nuestra institución para identificar variables de manejo susceptibles de modificación.

\footnotetext{
Correspondencia:

Christian José Pallares, Calle 4B № 27-97, apartamento 904, Edificio San Fernando del Viento, Cali, Colombia

Teléfono: (317) 4268197

icako@hotmail.com

Recibido: 15/05/13; aceptado: 27/11/13
}

\section{Materiales y métodos \\ Tipo de estudio, población e inclusión}

Entre enero y diciembre del 2011, se llevó a cabo un estudio prospectivo de cohortes en una institución de tercer nivel de complejidad en Cali. Se incluyeron todos los pacientes con diagnóstico de infecciones asociadas a la atención en salud en las unidades de cuidados intensivos y salas de hospitalización de acuerdo con las definiciones establecidas por los Centers for Disease Control and Prevention (CDC) de los Estados Unidos (14).

La inclusión de los pacientes se hizo de forma consecutiva, captándolos mediante el sistema de vigilancia activa de la infección implementado por el comité de infecciones de la institución, y el seguimiento se realizó desde el ingreso hospitalario hasta el egreso o la muerte. Se excluyó a quienes no tuvieran cultivo microbiológico asociado a la infección o reingresos hospitalarios en el curso de un año.

\section{Variables del estudio}

Se incluyeron variables sociodemográficas (sexo, procedencia, edad), clínicas (diagnóstico de ingreso, tipo y número de infecciones asociadas a la atención en salud, antecedentes de cirugía) y microbiológicas (tipo de bacteria según la coloración de Gram, microorganismo productor de betalactamasas de espectro extendido (BLEE+), microorganismo resistente a quinolonas, cefalosporinas de tercera generación o carbapenémicos, infección causada por Escherichia coli, Enterobacter cloacae o Proteus mirabilis (BLEE+), Staphylococcus aureus resistente a la oxacilina, Klebsiella pneumoniae (BLEE+), Acinetobacter baumannii resistente a carbapenémicos, Pseudomonas aeruginosa multirresistente o Enterococcus faecium resistente a vancomicina) (ESKAPE).

La variable de tratamiento antibiótico se definió como adecuada o inadecuada de acuerdo con lo establecido en la guía institucional de uso de antibióticos. Esta guía plantea un manejo empírico inicial de la infección según el perfil microbiológico hospitalario y hace parte de la estrategia de uso 
regulado de antibióticos implementada en la institución (15). En los casos con resultados de cultivos microbiológicos, el tratamiento antibiótico se determinó como adecuado o inadecuado según el perfil de sensibilidad bacteriana reflejado en el antibiograma. Se definió como antibiótico genérico o copia a todo fármaco que, con marca registrada o sin ella, no fuera el medicamento innovador para un determinado principio activo.

La variable de desenlace fue la muerte durante la estancia hospitalaria. El seguimiento a la supervivencia de los sujetos terminaba una vez egresaban del hospital.

\section{Plan de análisis estadístico}

El análisis estadístico descriptivo se realizó estableciendo proporciones para todas las variables. Se hizo un análisis de supervivencia para analizar el tiempo hasta el evento. Luego se practicó una prueba de rangos logarítmicos con el fin de comparar las funciones de supervivencia en las categorías de las variables. Para el análisis bivariado se estimó el riesgo relativo $(R R)$ y se ajustó un modelo multivariado de regresión de Cox con todas las variables para determinar la razón del riesgo de muerte (hazard ratio, HR). Los valores de $p$ menores de 0,05 se consideraron estadísticamente significativos.

\section{Consideraciones éticas}

Este estudio se clasificó como de riesgo mínimo de acuerdo con la Resolución 8430 de 1993 del Ministerio de Salud y fue aprobado por el comité de ética e investigación de la institución donde se realizó. Toda la información obtenida de los sujetos fue manejada exclusivamente por los investigadores y se le asignó a cada sujeto un código numérico para proteger su privacidad.

\section{Resultados}

Desde enero hasta diciembre del 2011 se incluyeron en el estudio 1.015 pacientes con diagnóstico de infecciones asociadas a la atención en salud. Las características sociodemográficas, clínicas, microbiológicas y de tratamiento se presentan en el cuadro 1.

El diagnóstico de ingreso más prevalente fue el trauma (heridas por arma de fuego, politraumatismo y quemaduras, $21 \%$, y los tumores sólidos, $11 \%$ ). La infección asociada a la atención en salud prevalente fue la de sitio operatorio (54\%), seguida por la asociada a dispositivos (catéter venoso y sonda vesical, $38 \%$ ). Del total de las bacterias
Cuadro 1. Características sociodemográficas, clínicas, microbiológicas y de tratamiento en los pacientes con diagnóstico de infecciones asociadas a la atención en salud, Hospital Universitario del Valle, 2011 ( $\mathrm{N}=1.015)$

\begin{tabular}{|c|c|c|}
\hline Características & $\mathbf{n}$ & $\%$ \\
\hline \multicolumn{3}{|l|}{ Sociodemográficas } \\
\hline \multicolumn{3}{|l|}{ Sexo } \\
\hline Masculino & 579 & 57 \\
\hline Femenino & 436 & 43 \\
\hline \multicolumn{3}{|l|}{ Procedencia } \\
\hline Cali & 607 & 60 \\
\hline Fuera de Cali & 382 & 38 \\
\hline Fuera del Valle & 26 & 3 \\
\hline \multicolumn{3}{|l|}{ Edad (años) } \\
\hline$<1$ & 42 & 4 \\
\hline $1-7$ & 48 & 5 \\
\hline $8-14$ & 34 & 3 \\
\hline $15-25$ & 134 & 13 \\
\hline $26-36$ & 139 & 12 \\
\hline $37-47$ & 185 & 18 \\
\hline $48-59$ & 170 & 17 \\
\hline $60-70$ & 95 & 10 \\
\hline$>70$ & 168 & 16 \\
\hline \multicolumn{3}{|l|}{ Clínicas } \\
\hline \multicolumn{3}{|l|}{ Diagnóstico de ingreso } \\
\hline Clínico & 477 & 47 \\
\hline Quirúrgico & 538 & 53 \\
\hline Infección del sitio operatorio & 550 & 54 \\
\hline Infección urinaria con sonda vesical & 186 & 18 \\
\hline Bacteriemia con dispositivo invasivo & 137 & 14 \\
\hline Neumonía con respiración mecánica & 59 & 6 \\
\hline Neumonía hospitalaria & 64 & 6 \\
\hline Cirugía previa a la infección & 121 & 12 \\
\hline \multicolumn{3}{|l|}{ Número de infecciones } \\
\hline 1 & 339 & 33 \\
\hline 2 & 252 & 25 \\
\hline 3 & 145 & 1 \\
\hline$>3$ & 279 & 28 \\
\hline \multicolumn{3}{|l|}{ Microbiológicas } \\
\hline Aislamiento de BGN & 633 & 62 \\
\hline Aislamiento positivo de BGN (BLEE+) & 155 & 15 \\
\hline Bacteria BGN resistente a quinolonas & 191 & 19 \\
\hline \multicolumn{2}{|l|}{ Bacteria BGN resistente a cefalosporinas } & 11 \\
\hline Bacteria BGN resistente a carbapenémicos & 31 & 3 \\
\hline Germen ESKAPE & 312 & 31 \\
\hline \multicolumn{3}{|l|}{ Tratamiento } \\
\hline \multicolumn{3}{|l|}{ Tratamiento antibiótico } \\
\hline Adecuado & 820 & 81 \\
\hline Inadecuado & 195 & 19 \\
\hline Uso de antibiótico genérico & 587 & 58 \\
\hline
\end{tabular}

BGN: bacilo Gram negativo

asociadas a la infección, $31 \%$ fue multirresistente, y entre ellas las más prevalentes fueron $A$. baumannii resistente a carbapenémicos (26\%), $K$. pneumoniae (BLEE+) (17\%), S. aureus resistente a la oxacilina (17\%) y E. coli (BLEE+) $(15 \%)$.

\section{Análisis de supervivencia y regresión de Cox}

La mortalidad global fue de $24,4 \%$. Los grupos de edad con mayor mortalidad fueron los de más de 70 años (25,4\%) y de 37 a 47 años (21,3\%). 
Se encontraron diferencias estadísticamente significativas para la mortalidad según edad $(p<0,001)$, diagnóstico de neumonía hospitalaria $(p=0,001)$, diagnóstico de ingreso quirúrgico $(p=0,010)$, presencia de los gérmenes ESKAPE $(p=0,026)$, terapia antibiótica inadecuada $(p<0,001)$ y uso de antibiótico genérico $(p<0,001)$ (figuras 1 a 6 y cuadro 2).

\section{Discusión}

Las infecciones asociadas a la atención en salud son un evento frecuente a nivel hospitalario. Algunos factores relacionados con su aparición no son modificables (enfermedades concomitantes, gravedad del cuadro clínico y edad), pero el tipo de infección adquirida puede estar influenciado por el manejo que recibe el paciente durante la hospitalización.

Según el modelo de análisis multivariado, en este estudio la probabilidad de morir de los sujetos con neumonía, comparada con la de aquellos sin este diagnóstico, fue superior a $60 \%$ y, aunque el diagnóstico de neumonía asociada a respiración mecánica asistida no fue estadísticamente significativo en el modelo bivariado, sí se comportó como un factor de riesgo. En la literatura se ha determinado una mortalidad asociada a esta enfermedad que varía entre 27 y $71 \%$ (16-19).

El riesgo de adquirir esta infección puede minimizarse con medidas de prevención como la higiene de manos, la elevación de la cabecera a $30-45^{\circ}$, los enjuagues orales con clorhexidina, el uso de un protocolo de sedación y destete, la aspiración de secreciones subglóticas y no cambiar los circuitos del respirador de manera rutinaria
(20). Estas medidas deben implementarse de manera integral, realizando seguimientos periódicos para garantizar su cumplimiento. El tratamiento antibiótico adecuado también ha demostrado ser un factor en la reducción de la mortalidad por neumonía (21-23). Esta variable se asoció en nuestros resultados con una probabilidad de morir dos veces mayor comparada con la de los sujetos que recibieron el tratamiento antibiótico de acuerdo con las guías institucionales de manejo de la infección.

Aunque existen múltiples formas de abordar el control de la resistencia bacteriana y el uso apropiado de antibióticos $(24,25)$, la caracterización de la infección con base en la ecología bacteriana, los patrones de resistencia y las infecciones más prevalentes permite crear estrategias específicas de control epidemiológico que garanticen una reducción del uso inapropiado de antibióticos y la resistencia bacteriana (15).

La mortalidad por estancia en las unidades de cuidados intensivos en países desarrollados oscila entre 7 y $21 \%$ (26). En este estudio, la probabilidad de morir de los sujetos que ingresaron a la unidad de cuidados intensivos fue de $50 \%$, casi $20 \%$ más que la reportada por otros estudios en el país $(27,28)$. Este factor se ha comportado como factor predictor independiente de la mortalidad en otros estudios (29-31), pero es importante mencionar que se ha demostrado que la presencia concomitante de infecciones asociadas a la atención en salud incrementa este riesgo significativamente (32). También se debe considerar que en el presente estudio el deterioro clínico de los pacientes en la unidad de

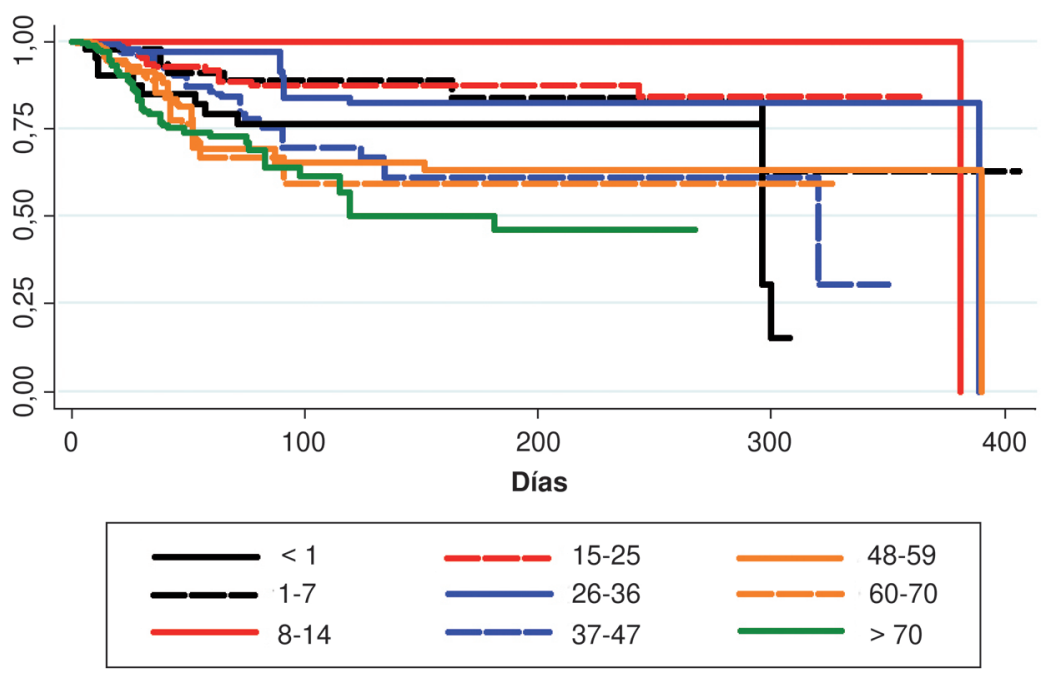

Figura 1. Supervivencia según edad (años), Hospital Universitario del Valle, 2011 


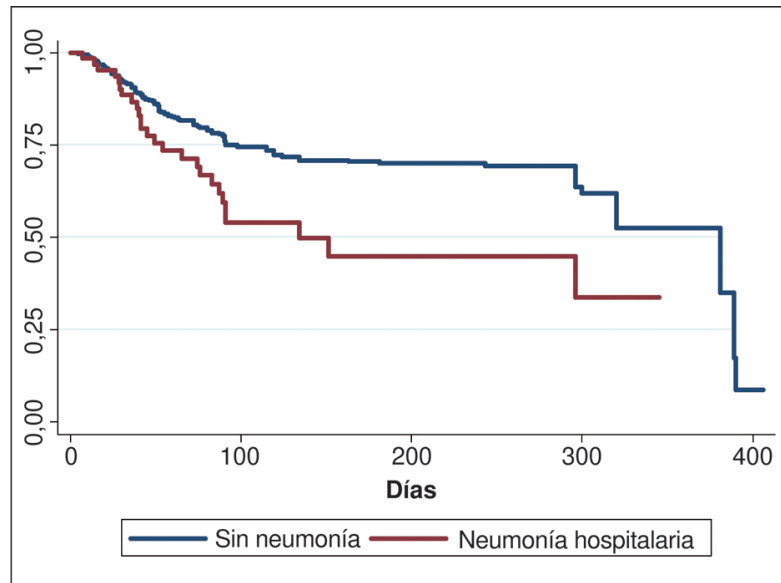

Figura 2. Supervivencia según diagnóstico de neumonía hospitalaria, Hospital Universitario del Valle, 2011

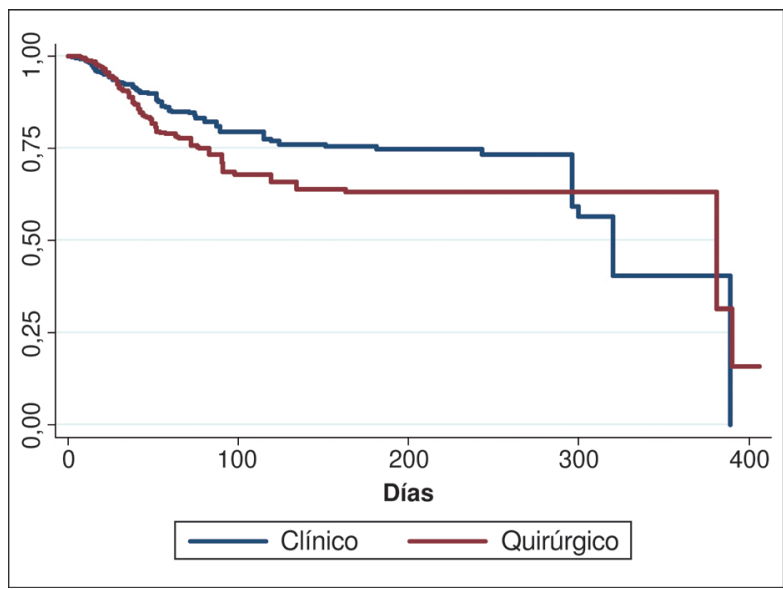

Figura 3. Supervivencia según diagnóstico de ingreso hospitalario Hospital Universitario del Valle, 2011

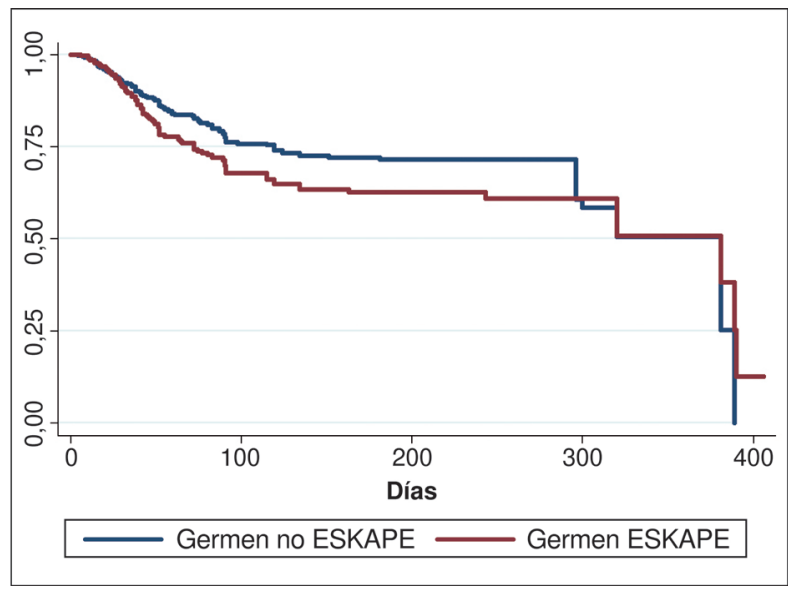

Figura 4. Supervivencia según presencia de gérmenes ESKAPE, Hospital Universitario del Valle, 2011

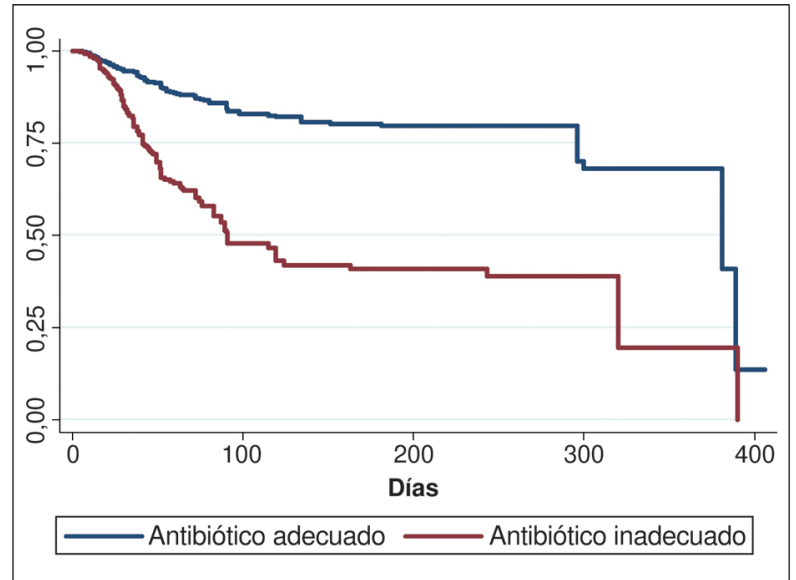

Figura 5. Supervivencia según tratamiento antibiótico, Hospital Universitario del Valle, 2011

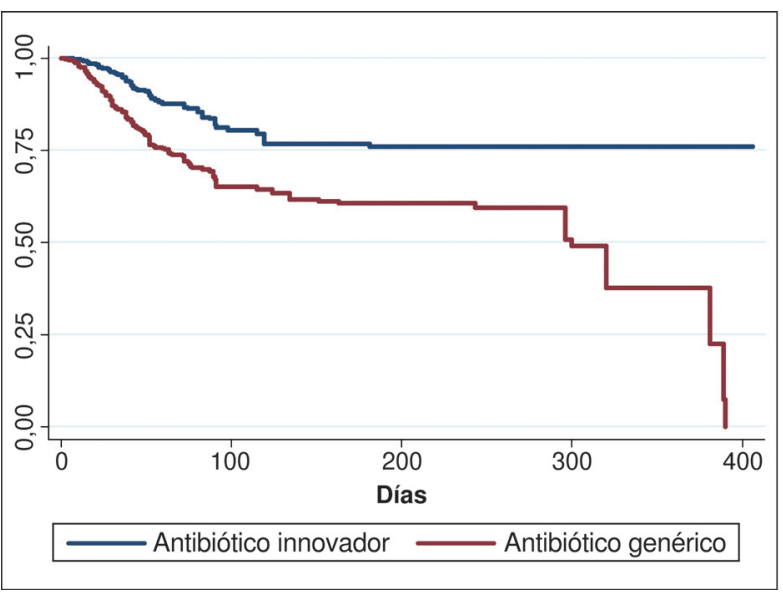

Figura 6. Supervivencia según antibiótico usado, Hospital Universitario del Valle, 2011

cuidados intensivos no se midió ni se estratificó con ninguna escala de gravedad, razón por la cual la mortalidad encontrada también puede atribuirse a causas relacionadas con el manejo y la condición de los sujetos hospitalizados en la unidad de cuidados intensivos.

En la práctica clínica en nuestro país existen diferentes alternativas de tratamiento de infecciones con antibióticos que responden a un mismo principio activo. Los productos llamados genéricos, moléculas que tienen una composición similar a las innovadoras, son las alternativas más empleadas, pues su precio de venta es menor al no requerir inversión en investigación, desarrollo y promoción. Existen datos de que estas moléculas difieren de las innovadores en equivalencia química (principio 
Cuadro 2. Factores de riesgo relacionados con la mortalidad por infecciones asociadas a la atención en salud, Hospital Universitario del Valle, $2011(\mathrm{~N}=1.015)$

\begin{tabular}{|c|c|c|c|c|}
\hline Variable & $\mathbf{R R}$ & $I \mathbf{C}_{95 \%}$ & HR & $I \mathbf{C}_{95 \%}$ \\
\hline Sexo & 1,17 & $0,94-1,45$ & $\ldots$ & $\ldots$ \\
\hline \multicolumn{5}{|l|}{ Edad (años) } \\
\hline$<1$ & 1,12 & $0,54-2,31$ & $\ldots$ & $\ldots$ \\
\hline $1-7$ & 0,49 & $0,21-1,13$ & $\ldots$ & $\ldots$ \\
\hline $8-14$ & 0,15 & $0,36-0,67$ & $\ldots$ & $\ldots$ \\
\hline $15-25$ & 0,32 & $0,17-0,58$ & $\ldots$ & $\ldots$ \\
\hline $26-36$ & 0,32 & $0,17-0,59$ & $\ldots$ & $\ldots$ \\
\hline $37-47^{*}$ & & & $\ldots$ & $\ldots$ \\
\hline $48-59$ & 0,95 & $0,59-1,51$ & 3,59 & $1,46-3,67$ \\
\hline $60-70$ & 1,20 & $0,70-2,05$ & 3,12 & $1,34-3,57$ \\
\hline$>70$ & 1,49 & $0,96-2,33$ & 3,22 & $1,29-2,85$ \\
\hline Diagnóstico de ingreso & 1,33 & $1,07-1,67$ & $\ldots$ & $\ldots$ \\
\hline Infección de herida quirúrgica & 0,93 & $0,74-1,15$ & $\ldots$ & $\ldots$ \\
\hline Bacteriemia asociada a catéter & 1,23 & $0,92-1,64$ & $\ldots$ & $\ldots$ \\
\hline Infección urinaria asociada a sonda vesical & 0,78 & $0,57-1,06$ & $\ldots$ & $\ldots$ \\
\hline Neumonía hospitalaria & 1,74 & $1,26-2,39$ & 1,66 & $1,09-2,52$ \\
\hline Neumonía por respiración mecánica asistida & 1,49 & $1,04-2,15$ & $\ldots$ & $\ldots$ \\
\hline Antecedentes de cirugía previa al diagnóstico de infección & 0,79 & $0,54-1,15$ & $\ldots$ & $\ldots$ \\
\hline Infección por microorganismo ESKAPE & 1,47 & $1,18-1,83$ & $\ldots$ & $\ldots$ \\
\hline Infección por microorganismo Gram negativo & 1,27 & $1,00-1,60$ & $\ldots$ & $\ldots$ \\
\hline Infección por microorganismo BLEE+ & 1,07 & $0,79-1,43$ & $\ldots$ & $\ldots$ \\
\hline Infección por bacteria resistente a ciprofloxacina & 0,98 & $0,74-1,29$ & $\ldots$ & $\ldots$ \\
\hline Infección por bacteria resistente a meropenem & 1,33 & $0,79-2,25$ & $\ldots$ & $\ldots$ \\
\hline Infección por bacteria resistente a ceftriaxona & 0,95 & $0,64-1,31$ & $\ldots$ & $\ldots$ \\
\hline Estancia en unidad de cuidados intensivos & 2,16 & $1,76-2,67$ & 1,50 & $1,13-1,99$ \\
\hline Uso inadecuado de antibióticos & 1,62 & $1,29-2,04$ & 3,06 & $2,34-3,99$ \\
\hline Uso de antibiótico genérico o copia & 2,19 & $1,69-2,83$ & 1,93 & $1,44-2,57$ \\
\hline Presencia de 2 o más IAAS & 1,89 & $1,43-2,50$ & $\ldots$ & $\ldots$ \\
\hline
\end{tabular}

* Edad de referencia; RR: riesgo relativo; HR: cociente de riesgos; IAAS: infecciones asociadas a la atención en salud

activo), potencia (actividad antibacteriana in vitro) y biodisponibilidad (concentraciones del antibiótico en plasma y tejidos) $(33,34)$.

Tambien se han llevado a cabo comparaciones en modelos animales que han demostrado cómo la actividad antibacteriana es inferior en varios productos genéricos (35-37), y existen reportes en la literatura sobre fallas terapéuticas de dichas moléculas (38).

Estas situaciones podrían explicar hasta cierto punto los hallazgos del presente estudio, en el cual la probabilidad de morir de los pacientes que recibieron antibióticos genéricos fue casi dos veces mayor que la de quienes recibieron un antibiótico innovador. Además, en la literatura científica no se registra ningún antibiótico genérico de los empleados en nuestro país que haya sido probado por medio de ensayos clínicos controlados para establecer su eficacia clínica y seguridad, y, sin embargo, se ha asumido que son las mismas del producto innovador. La eficacia clínica no solo depende del principio activo sino de la tecnología farmacéutica (definida como los procesos de manufactura del antibiótico), la cual puede ser responsable de reducir o eliminar propiedades del fármaco, alterando su mecanismo de acción (39).

Bajo esta premisa, el uso de antibióticos no innovadores podría considerarse arriesgado, principalmente por el eventual daño a la salud del paciente debido a una falla terapéutica, así como por el costo económico para el paciente o el sistema de salud resultado de la necesidad de cuidados adicionales, por las implicaciones sociales y emocionales ante el fracaso en la resolución de la enfermedad infecciosa y por la potencial pérdida de la confianza en el médico al no lograr una mejoría en la salud del paciente.

Este trabajo identifica estrategias susceptibles de modificar ciertas condiciones con el fin de reducir la mortalidad en los pacientes con infecciones asociadas a la atención en salud. Dichas estrategias incluyen el uso adecuado de antibióticos, lo que se logra a través de programas de vigilancia, control, protocolización e intervención en el uso de 
antibióticos (antibiotic stewardship), y el empleo de antibióticos que garanticen una respuesta terapéutica equivalente a la de las moléculas innovadoras.

\section{Agradecimientos}

Deseamos agradecer la colaboración del equipo de trabajo de epidemiología hospitalaria del Comité de Infecciones y del Laboratorio de Microbiología del Hospital Universitario del Valle "Evaristo García", E.S.E.

\section{Conflicto de intereses}

Los autores declaran no tener ningún conflicto de intereses en la realización del estudio.

\section{Financiación}

El presente estudio fue financiado con recursos propios del Hospital Universitario del Valle "Evaristo García", E.S.E., y no tuvo financiación de parte de la empresa privada.

\section{Referencias}

1. Organización Mundial de la Salud. Iniciativa mundial en pro de la seguridad del paciente y directrices sobre higiene de las manos en la atención sanitaria. Geneva: WHO; 2005.

2. Cataño JC, Castaño O. Evaluación de un programa de vigilancia epidemiológica del consumo de antibióticos y la flora en una clínica de tercer nivel. Infectio. 2009;13:6-13.

3. Rekha M. Implementation of strategies to control antimicrobial resistance. Chest. 2001;119:405S-11S. http://dx.doi.org/10. 1378/chest.119.2_suppl.405S

4. Olaechea PM, Ulibarrena MA, Álvarez-Lerma F, Insausti J, Palomar M, De la Cal MA. Factors related to hospital stay among patients with nosocomial infection acquired in the intensive care unit. Infect Control Hosp Epidemiol. 2003:24:207-13. http://dx.doi.org/10.1086/502191

5. DiGiovine B, Chenoweth C, Watts C, Higgins M. The attributable mortality and costs of primary nosocomial bloodstream infections in the intensive care unit. Am J Respir Crit Care Med. 1999;160:976-81. http://dx.doi.org/10.1164/ ajrccm.160.3.9808145

6. Pittet D, Tarara D, Wenzel RP. Nosocomial bloodstream infection in critically ill patients. Excessive length of stay, extra costs, and attributable mortality. JAMA. 1994;271:1598-601. http://dx.doi.org/10.1001/jama.1994.03510440058033

7. Sostarich AM, Zolldann D, Haefner $H$, Luetticken $\mathbf{R}$, Schulze-Roebecke $\mathbf{R}$, Lemmen SW. Impact of multiresistance of Gram-negative bacteria in bloodstream infection on mortality rates and length of stay. Infection. 2008;36:31-5. http://dx.doi.org/10.1007/s15010-007-6316-4

8. Renaud B, Brun-Buisson C. Outcomes of primary and catheter-related bacteremia. A cohort and case-control study in critically ill patients. Am J Respir Crit Care Med. 2001;163: 1584-90. http://dx.doi.org/10.1164/ajrccm.163.7.9912080

9. Fabbro-Peray P, Sotto A, Defez C, Cazaban M, Molinari $\mathbf{L}$, Pinede $\mathbf{M}$, et al. Mortality attributable to nosocomial infection: A cohort of patients with and without nosocomial infection in a French university hospital. Infect Control Hosp Epidemiol. 2007;28:265-72.

10. Blot S. Limiting the attributable mortality of nosocomial infection and multidrug resistance in intensive care units. Clin Microbiol Infect. 2008;14:5-13. http://dx.doi.org/10.1111/ j.1469-0691.2007.01835.x

11. Garnacho-Montero J, Ortíz-Leyba C, Herrera-Melero I, Aldabo-Pallas T, Cayuela-Domínguez A, MárquezVacaro JA, et al. Mortality and morbidity attributable to inadequate empirical antimicrobial therapy in patients admitted to the ICU with sepsis: A matched cohort study. J Antimicrob Chemother. 2008; 61:436-41. http://dx.doi. org/10.1093/jac/dkm460

12. Cosgrove SE. The relationship between antimicrobial resistance and patient outcomes: Mortality, length of hospital stay, and health care costs. Clin Infect Dis. 2006;42:S82-9. http://dx.doi.org/10.1086/499406

13. McGregor JC, Rich SE, Harris AD, Perencevich EN, Osih $\mathbf{R}$, Lodise TP, et al. A systematic review of the methods used to assess the association between appropriate antibiotic therapy and mortality in bacteremic patients. Clin Infect Dis. 2007; 45:329-37. http://dx.doi.org/10.1086/519283

14. Horan TC, Gaynes RP. Surveillance of IASC Infections. Hosp Epidemiol Infect Control. 2004;1659-702.

15. Pallares CJ, Martínez E. Implementación de un programa de uso regulado de antibióticos en dos unidades de cuidado intensivo médico-quirúrgico en un hospital universitario de tercer nivel en Colombia. Infectio. 2012;16:192-8.

16. Cook DJ, Walter SD, Cook RJ, Griffith LE, Guyatt GH, Leasa. D, et al. Incidence of and risk factors for ventilator associated pneumonia in critically ill patients. AVAP Intern Med. 1998; 129: 433-40. http://dx.doi.org/10.7326/00034819-129-6-199809150-00002

17. Leone M, Garcin F, Bouvenot J, Boyadjev I, Visintini $\mathbf{P}$, Albanese $\mathbf{J}$, et al. Ventilator associated pneumonia: Breaking the vicious circle of antibiotic overuse. Crit Care Med. 2007;35:379-85. http://dx.doi.org/10.1097/01.CCM. 0000253404.69418.AA

18. Fagon JY, Chastre J, Vuagnat A, Trouillet JL, Novara A, Gibert C. Nosocomial pneumonia and mortality among patients in intensive care units. JAMA. 1996;275:866-9. http://dx.doi.org/10.1001/jama.1996.03530350048033

19. Tejada A, Bello S, Chacón E, Muñoz J, Villuendas MC, Figueras $\mathbf{P}$, et al. Risk factors for nosocomial pneumonia in critically ill trauma patients. Crit Care Med. 2001;29:304-9.

20. Ali A, Tous A, De Vivero A, Hernández A, Alarcón AM, Leal AL, et al. Consenso Colombiano de Neumonía Nosocomial 2013. Acta Colombiana de Cuidado Intensivo. 2013;13:46-135

21. Dotson RG, Pingleton SK. The effect of antibiotic therapy on recovery of intracellular bacteria from bronchoalveolar lavage in suspected ventilator associated nosocomial pneumonia. Chest. 1993;103:541-6. http://dx.doi.org/10. 1378/chest.103.2.541

22. The Canadian Clinical Care Trials Group. A randomized trial of diagnostic techniques for ventilator-associated pneumonia. N Engl J Med. 2006;355:2619-30. http://dx.doi. org/10.1056/NEJMoa052904 
23. Fagon Jy, Chastre J, Wolff M, Gervais C, Parer-Aubas $\mathrm{S}$, Stéphan $\mathrm{F}$, et al. Invasive and noninvasive strategies for management of suspected ventilator-associated pneumonia. A randomised trial. Ann Intern Med. 2000; 132: 621-30. http://dx.doi.org/10.1056/NEJMoa052904

24. Bantar C, Vesco E, Heft C, Salamone F, Krayeski M, Gómez $\mathbf{H}$, et al. Replacement of broad-spectrum cephalosporins by piperacillin-tazobactam: Impact on sustained high rates of bacterial resistance. Antimicrob Agents Chemother. 2004;48:392-5. http://dx.doi.org/10.1128/AAC.48.2.392-395.2004

25. Dellit TH, Owens RC, McGowan JE Jr, Gerding DN, Weinstein RA, Burke JP, et al. Infectious Diseases Society of America; Society for Healthcare Epidemiology of America. Infectious Diseases Society of America and the Society for Healthcare Epidemiology of America guidelines for developing an institutional program to enhance antimicrobial stewardship. Clin Infect Dis. 2007;44:159-77. http://dx.doi. org/10.1086/510393

26. World Health Organization. Manual of the international statistical classification of diseases, injuries and causes of death. Geneva: WHO; 1977.

27. Pérez JD, Rodríguez García LC, Alcalá-Cerra G. Mortalidad e infecciones nosocomiales en dos unidades de cuidados intensivos de la ciudad de Barranquilla (Colombia). Salud UniNorte. 2008;24:74-86.

28. Pérez A, Dennis R, Rondón M, Metcalfed M, Rowane K. A Colombian survey found intensive care mortality ratios were better in private vs public hospitals. J Clin Epidemiol 2006;59:94-101. http://dx.doi.org/10.1016/j.jclinepi. 2005.06.004

29. Averill R, Goldfield N, Hughes J, Bonazalli J. All patients refined Diagnosis Related Groups (APR-DRGs) Methodology Overview. Version 2.0. Wallingford: 3M Health Information Systems, 2003. p.1-91.

30. Society of Critical Care Medicine. History. Mount Prospect, SCCM; 2012. Fecha de consulta: 1 de julio de 2013. Disponible en: http://www.sccm.org/About-SCCM/Pages/ Governance.aspx.

31. Horan T, Andrus M, Dudeck M. CDC/NHSN surveillance definition of health-care associated infection and criteria for specific types of infections in the acute care setting. Am J Infect Control 2008;36:309-32. http://dx.doi.org/10.1016/j. ajic.2008.03.002

32. Meléndez HJ, Naranjo FF, Franco DL, Carvajal T. Mortalidad general y atribuible a cuidado intensivo: estudio de cohortes. Acta Colombiana de Cuidado Intensivo. 2011; 11:91-9.

33. Jones $\mathbf{R}$, Fritsche $\mathbf{T}$, Moet $\mathbf{G}$. In vitro potency evaluations of various piperacillin/tazobactam generic products compared with the contemporary branded (Zosyn, Wyeth) formulation. Diagn Microbiol Infect Dis. 2007;65:319-22. http://dx.doi. org/10.1016/j.diagmicrobio.2007.12.010

34. Nightingale $\mathbf{C H}$. A survey of the quality of generic clarithromycin products manufactured in Slovenia and Israel. Adv Ther. 2000;17:167-78.

35. Zuluaga A, Agudelo M, Cardeño J, Rodríguez C, Vesga 0 . Determination of therapeutic equivalence of generic products of gentamicin in the neutropenic mouse thigh infection model. PLOS One. 2010;5:1-11. http://dx.doi.org/10. 1371/journal.pone.0010744

36. Rodríguez C, Agudelo M, Zuluaga A, Vesga O. In vitro and in vivo comparison of the anti-staphylococcal efficacy of generic products and the innovator of oxacillin. BMC Infect Dis. 2010;10:153. http://dx.doi.org/10.1186/1471-2334-10-153

37. Vesga O, Agudelo M, Salazar B, Rodríguez C, Zuluaga A. Generic vancomycin products fail in vivo despite being pharmaceutical equivalents of the innovator. Antimicrob Agents Chemother. 2010;54:3271-9. http://dx.doi.org/10. 1128/AAC.01044-09

38. Rodríguez CA, Agudelo M, Cataño JC, Zuluaga A, Vesga O. Potential therapeutic failure of generic vancomycin in a liver transplant patient with MRSA peritonitis and bacteriemia. J Infect. 2009;59:277-80. http://dx.doi.org/10. 1016/j.jinf.2009.08.005

39. Lehr H, Brunner J, Rangonwala R, Kirkpatrik C. Particulate matter contamination of intravenous antibiotics aggravates loss of functional capillary density of postischemic striated muscle. Am J Respir Crit Care Med. 2002; 165:514-20. http://dx.doi.org/10.1164/ajrccm.165.4.2108033 